\title{
The comparative effects of intermittent versus continuous energy restriction on postprandial glucose-lipid metabolism following $5 \%$ weight-loss: interim analysis of an ongoing study
}

\author{
R. Antoni ${ }^{1}$, K.L. Johnston ${ }^{2}$, A.L. Collins ${ }^{1}$ and M.D. Robertson ${ }^{1}$ \\ ${ }^{1}$ Nutrition, Metabolism and Diabetes Research Group, Faculty of Health and Medical Sciences, University of Surrey, \\ Guildford, GU2 7WG, UK and ${ }^{2}$ Lighterlife UK Ltd, Cavendish House, Parkway, Harlow Business Park, Essex, CM19 \\ $5 Q F, U K$
}

The intermittent energy restriction (IER) approach to weight-loss involves short periods of substantial (>70 \%) energy restriction (ER) interspersed with normal eating ${ }^{(1)}$. Previous work has demonstrated the capacity of substantial ER to elicit acute, weight-loss independent alterations to fasting and postprandial glucose-lipid metabolism in a dose response manner ${ }^{(2)}$ The present study aims to build on our earlier work by characterising the metabolic, physiological and anthropometric changes that occur during IER, and to compare this to weight-loss achieved through a more modest, continuous energy restriction (CER).

18 (11 male) overweight/obese participants (46 $\left.\pm 3 \mathrm{y}, \mathrm{BMI}: 30 \cdot 1 \pm 1 \cdot 0 \mathrm{~kg} / \mathrm{m}^{2}\right)$ were randomised to either an intermittent $(2638 \mathrm{~kJ}$ for 2 consecutive days/week, $\mathrm{n}=9$ ) or continuous $(2510 \mathrm{~kJ}$ below requirements) ER diet group. 6-hour postprandial responses to a test meal, lipid profiles, anthropometry and blood pressure (BP) were assessed at baseline and following attainment of a $5 \%$ weight-loss. Data were analysed using independent t-tests (for between-group comparisons) and paired t-tests (for within-group comparisons), with results presented as mean \pm SEM.

Time to attain a $5 \%$ weight loss was similar between IER and CER diet groups $(60 \pm 7$ days and $72 \pm 12$ days respectively, $\mathrm{p}=$ 0.403). Weight-loss was accompanied by comparable reductions in fat mass, waist and hip circumference in both groups ( $p$ value: within group all $p<0.05$; between groups all $p>0.05$ ). Following weight-loss, trends in favour of a reduction in incremental triacylglycerol responses was observed in the IER group only ( $p$ value: within-group $p=0 \cdot 085$; between-groups $p=0 \cdot 271$ ), as well as between-group differences in the change from baseline of incremental glucose responses $(p=0.075)$. Neither diet significantly influenced fasting lipid profiles, although systolic BP was reduced following IER only $(\mathrm{p}=0 \cdot 007)$.
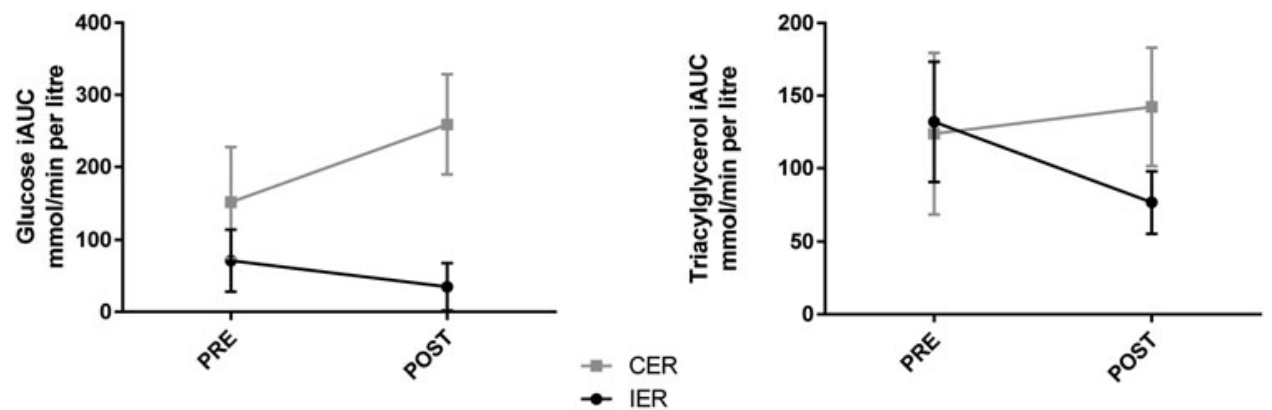

Postprandial responses pre and post $5 \%$ weight-loss. iAUC: incremental area under curve.

Our interim data suggests that a modest $5 \%$ weight-loss through IER may potentially exert more favourable effects on postprandial glucose-lipid metabolism when compared to similar weight-loss achieved through CER. Changes in all other metabolic, physiological and anthropometric outcomes were mostly comparable. Data presented here are taken from an interim analysis of an ongoing study. Analysis from the final study cohort is required before firm conclusions can be drawn. Additional analyses are also planned, which may yield mechanistic insights behind any observed differences.

1. Antoni R, Johnston KL, Collins AL et al. (2014) Res Endocrinol 2014 Article ID 459119

2. Antoni R, Johnston KL, Collins AL et al. (2016) Br J Nutr 115(6), 951-959 